\title{
Sprachen lernen und lehren \\ ist sprachenpolitisch handeln
}

Gedanken und Informationen zum UNESCO-Projekt "LINGUAPAX»

\author{
Prop. Dr. AlbERT RAasch \\ Romanistisches Institut, \\ Universität des Saarlandes, \\ Saarbrücken
}

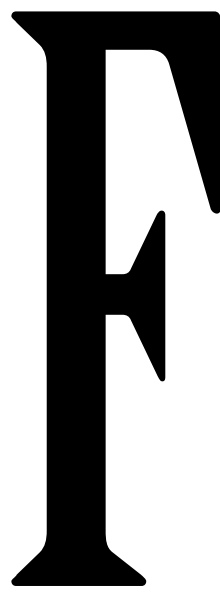

rieden, peace, pax, eirene, rauha...

«LINGUAPAX»: Bei erstem Hinsehen scheint sich die Bedeutung wie selbstverständlich selbst zu erklären: «Frieden durch Sprache»; der Ausdruck erscheint wie ein Aufruf, der selbstverständlicher und überzeugender nicht sein könnte - bei erstem Hinsehen. «Frieden» ist die vertraute Übersetzung von «pax»; jedes einschlägige Wörterbuch bestätigt dies. Aber: In dem Wort «Frieden» steckt (ursprünglich) eine Bedeutung, die sich durch die Verwandtschaft mit Wörtern wie "frei» und seine Ableitungen (und auch "Freude», "froh») andeutend erläutern lässt. Dagegen "pax»: verwandt mit "pangere», genauer mit dessen Partizip Perfekt «pactum», aus dem schon ersichtlich wird, wie "pax» zustande kommt, nämlich durch Vertrag oder Vereinbarung oder Festlegung. Die Etymologie erlaubt eine erste Annäherung; sie lässt sich ergänzen durch die Semantik. Wenn man sagt "den Frieden stören» oder «brechen», dann ist Frieden ein Zustand; «Frieden schließen» legt nahe, dass damit eine Abmachung, ein Abkommen gemeint ist. Ein Friedensschluss muss nicht Frieden bringen oder bedeuten oder meinen: So also kann man den Unterschied zwischen diesen beiden Bedeutungen auf den Punkt bringen. Die Frage ist also berechtigt: Was ist mit «Linguapax» gemeint?

«Linguapax»: Frieden durch «Sprache»? Ist "Frieden» in dem sprachlichen System enthalten? Wie ist es dort enthalten und verlinkt? Bezeichnet es einen $\mathrm{Zu}$ - stand, etwas Seiendes, etwas Gewordenes, etwas Abgeschlossenes ? Gibt es vielleicht Sprachen, die den Begriff 'Frieden' differenzierter, nuancierter ausdrücken als andere, weil sie z.B. über reichere Ausdrucksmöglichkeiten für dieses begriffliche Feld verfügen? Einschlägige Wörterbücher bieten für «friedlich» z.B. im Französischen «paisible» und «pacifique» an; Synonyme? Ein finnisches Wörterbuch nennt für «rauha» verschiedene deutsche (synonyme ?) Adjektive: friedlich, ruhig, friedfertig, gelassen, ausgeglichen.

Es ergeben sich ganz andere Perspektiven, wenn ich «Sprache» nicht als System, sondern als «Rede», also als «Sprachverhalten» interpretiere. Der Zustand des Friedens könnte dann bewahrt (oder gestört) werden, wenn ich die Sprache friedfertig (oder provozierend) verwende, wenn ich über das (fehlende) Verstehen des anderen zur Verständigung mit dem anderen (nicht) komme; wenn ich

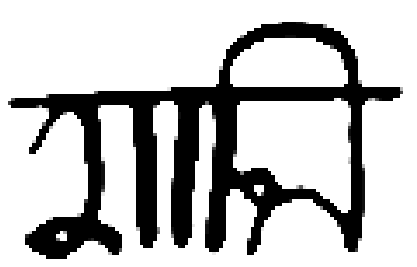

'Frieden' als Abwesenheit von 'Krieg'. Solange kein Friedensschluss vorliegt, ist der Kriegszustand nicht beendet. An der Siegfriedlinie sprach man von "une drôle de guerre», weil man - trotz Krieg - meinen konnte, im tiefsten Frieden zu leben.

Muss man (sich auf) den Krieg vorbereiten, wenn man Frieden haben/bewahren will? Es gibt schmerzliche Erfahrungen, dass diese sprichwörtliche Formulierung eine Lebensweisheit ausspricht; mit «si vis pacem, para pacem» möchte ich zum Ausdruck bringen, dass man, wenn man Frieden will, Frieden (stets von neuem) machen, erwerben, schaffen, im Sinne haben muss. Frieden ist also kein $\mathrm{Zu}$ stand der Passivität, ganz im Gegenteil: Frieden ist ein aktives Verhalten, ein Tun, eben auch mit/durch Sprache. Ich vermisse weiterhin im Deutschen (aber nicht nur dort) ein Verb wie «ich friede, du friedest, er/sie friedet, wir frieden...».

Damit sind wir in unseren Überlegungen an dem mich so ausdrücke, dass ich den Anderen (nicht) akzeptiere, ihn (nicht) respektiere, (nicht) partnerschaftlich mit ihm kommuniziere. Die Konsequenzen wären also recht unterschiedlich: nuanciertes Systemwissen fördern? oder nuanciertes sprachliches Verhalten fördern? Vielleicht ja beides, aber jedes von beiden verlangt unterschiedliche Anstrengungen beim Lernen und beim Lehren von Sprache.

\section{«Linguapax». ein Appell}

«Si vis pacem, para pacem»: So habe ich einmal einen Aufsatz betitelt, und damit wollte ich zweierlei zum Ausdruck bringen. 'Frieden ' und 'Krieg' sind zusammenhängende Begriffe; «Krieg und Frieden» als Buchtitel, im Lateinischen «pace belloque»;
Punkt angelangt, wo «lingua»/«Sprache» als Verhalten, besser als «Tun», als «Handeln» zu verstehen ist. «Friedenskompetenz» ist also «Handlungskompetenz»; jetzt wird deutlich, dass das Bemühen um «Frieden durch Sprache» koinzidiert mit dem Bemühen, Sprache als Handlung zu beschreiben. Womit wir bei der Linguistik, genauer: der Pragmalinguistik angekommen sind. "Linguapax» ist also nicht nur «Appell», sondern Programm, es ist ein Programm zu Definition der Zielsetzung, die man mit Sprachunterricht und mitSpracherwerb erreichen will, anders gesagt: «Linguapax» ist eine Philosophie. Dass wir eine «Erziehung zum Frieden» brauchen, dass aber der Weg von der Philosophie zum Handeln weit ist, hat Reinhold Freuden- 


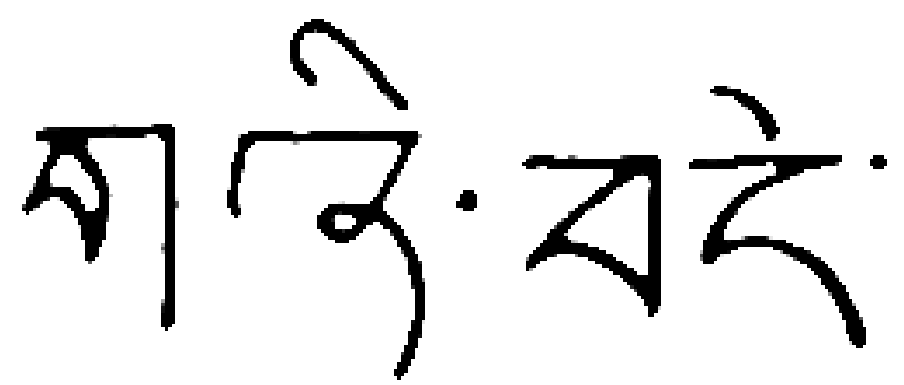

stein folgendermaßen ausgedrückt (Freudenstein 1997: 93)

«Das vom Autor erfahrene geringe Interesse an Seminaren zum Thema ,Erziehung zum Frieden' zeigt, daß zumindest deutsche Sprachstudenten/-innen und Sprachlehrer/-innen es nicht für nötig halten, die Friedensthematik im Studium und im Sprachunterricht zu behandeln.»

Er erklärt dieses Phänomen so:

«Da Frieden in den traditionellen Lernmaterialien unter allgemeinbildende Lernziele eingeordnet wird, fühlt sich kein Lehrer/keine Lehrerin für das Erreichen dieser Ziele persönlich verantwortlich. So hat die Vokabel, peace' oder ,paix‘, wenn sie im 3. oder 4. Lernjahr eingeführt wird, den gleichen Rang wie jede andere Vokabel, hinter der kein Konzept, keine Anziehungskraft steht.»

Die Umsetzung einer Philosophie des Friedens im Sprachunterricht ist also ein interdisziplinäres Problem; sie berührt die Erziehungswissenschaft, insbesondere die pädagogische Psychologie (Motivation, Lehrerrolle), die Fachdidaktik, die Linguistik, die Sprachenpolitik. Überlegungen und Einsichten dieser Art führten zu einem Programm und dann zu einem Projekt, bekannt geworden unter dem Namen «LINGUAPAX».

\section{LINGUAPAX - eine Philosophie, ein Programm, ein Projekt}

Ein Expertentreffen der Mitglieder des Internationalen LINGUAPAX-Komitees hat die Philosophie dieses Projektes beraten und genauer formuliert ${ }^{1}$. Hier sind die wichtigsten Gedanken in einer Zusammenfassung, die imübrigen zeigt, wie aktuell die Einschätzung der Situation auch heute ist: In der heutigen Welt, in der die Bezie- hungen zwischen den verschiedenen Ländern viel enger als früher geworden sind und in der gleichzeitig die Konflikte und Spannungen zwischen verschiedenen $\mathrm{Na}$ tionen und ethnischen Gruppen fortbestehen, gibt es ein vorrangiges und dringendes Bedürfnis, Mittel und Wege zu finden, wie die gefährliche Entwicklung der rassistischen und nationalistischen Gefühle, die Verbreitung von Hass, der Entfremdung vom Nächsten oder auch nur der Ignorierung des Nächsten, der Länder und Völker-benachbart oder weit entfernt voneinander - im Hinblick auf ihre geographi-

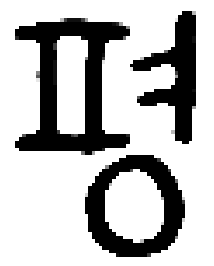
sche, kulturelle wirtschaftliche Situation vermieden werden kann. Seinen Nächsten besser zu kennen, ihn besser zu verstehen, um im Interesse aller besser mit ihm zusammenarbeiten zu können, ist der kategorische Imperativ unserer Gegenwart geworden. Dieses Problem kann und muß auf verschiedenen Wegen und mit verschiedenen Mitteln gelöst werden, und die Erziehung ist aufgerufen, einen wichtigen Beitrag dazu zu leisten, in der Welt ein Klima des Vertrauens, der Freundschaft, der gegenseitigen Verständigung zu schaffen. Eines der wirksamsten Mittel, um dieses Ziel zu erreichen, ist die Vermittlung und das Erlernen fremder Sprachen. Allerdings lernt man die Fremdsprachen bereits seit Jahrhunderten, und man macht immer wieder bittere Erfahrungen: Man lernt sie, um den Feind besser bekämpfen zu können. Man lernt sie, um die eigene wirtschaftliche Vor-

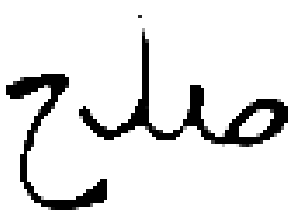
herrschaft zu festigen. Man lernt sie, um in seiner beruflichen Karriere mehr Erfolg zu haben usw. In Anbetracht der Herausforderungen un- serer Zeit müssen wir heute dem Fremdsprachenunterricht ein neues Lebens und einen moralischen Sinn geben.

Diese Überlegungen, die in Venedig 1989 formuliert wurden, gingen auf erste Beratungen zurück, die, bekannt als «LINGUAPAX I» 1987 in Kiew, der damaligen Sowjetrepublik Ukraine, stattfanden. Um die Offenheit der Perspektiven dieses ersten Seminars zu dokumentieren, sei dar-

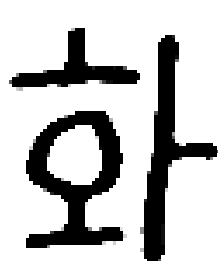
an erinnert, dass dort (nach meiner Kenntnis zum ersten Mal; A.R.) «an International Year of Languages» vorgeschlagen wurde ${ }^{3}$, eine Forderung, die dann ja schließlich 2001 er-

füllt wurde. Dieses erste Seminar in Kiew, ausgerichtet vom Germanistischen Institut der Staatlichen Universität Kiew, war eine internationale Zusammenkunft von Experten und Entscheidungsträgern; die Namen einiger Persönlichkeiten, die sicherlich vielen Lesern und Leserinnen bekannt sein dürften, sollen hier genannt werden: Prof. Ingrid Classen-Bauer (Universität Lüneburg), Prof. Stathis Efsthathiadis (Aristoteles-Universität, Thessaloniki), Prof. Claude Frioux (Université Paris VIII), Prof. Wil Knibbeler (Katholieke Universiteit Nijmegen), Dr. Brigitte Krück (Pädagogische Hochschule Potsdam), Prof. Oleg Semenets (Universität Kiew), Prof. Miguel Siguan (Universität Barcelona), Prof. György Szepe (Ungarische Akademie der Wissenschaften, Budapest), Prof. Elizabetta Zuanelli

(Universitá Ca'Foscari, Venedig). In der UNESCO war der damalige Leiter des LINGUAPAX-Projekts Prof. Victor Koptilov (Staatliche Universität Kiew, Germanistisches Institut). 
Viele dieser Persönlichkeiten nahmen dann an einem späteren, von uns ausgerichteten Seminar, nämlich LINGUAPAX III, in Saarbrücken teil, zusammen u.a. mit Prof. Marcel de Grève (Universität Brüssel, seinerzeit Präsident der AIMAV), Prof. Gert Hummel (Universität des Saarlandes, Saarbrücken, nach Eintritt in den Ruhestand Bischof der Evangelischen Gemeinde in Tbilissi), Frau Prof. Irmela Neu-Altenheimer (Fachhochschule München, seinerzeit Wissenschaftliche Mitarbeiterin der Deutschen UNESCO-Kommission, Bonn), Prof. Bernd Spillner (Gesamthochschule - Universität Duisburg, damals Präsident der Gesellschaft für Angewandte Linguistik - GAL), Prof. JeanPierre van Deth (Président du CIREEL, Paris), Prof. Diether Breitenbach, (Universität

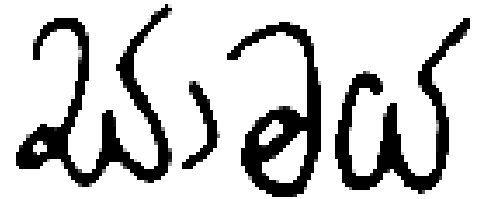
des Saarlandes, Saarbrücken, seinerzeit Kultusminister des Saarlandes) u.a.; andere Persönlichkeiten sind im Laufe der Jahre (bei den Veranstaltungen in Barcelona, Melbourne, Tokyo, Hiroshima usw.) hinzugetreten. Die Kontinuität und auch die Lebendigkeit des Projekts werden also

\section{rauha} nicht nur durch die grundlegende Philosophiegarantiert, sondern auch durch

die Personen, die diese Ideen tragen, sie in Projekte und Programme umsetzen und auf vielfältige Weise international über viele Jahre zusammenarbeiten.

Die Kontinuität zeigt sich auch in den grundlegenden LINGUAPAX-Seminaren, deren vollständige Liste so lautet: $1987 \mathrm{Kiew}$ (SU, Ukraine); 1989 Sitges (Spanien), 1990 Saarbrücken (Deutschland), 1994 Barcelona (Spanien), 1995 Melbourne (Australien). Diese Serie von Veranstaltungen und ihrer Dokumentationen verband die Zeit vor mit der Zeit nach der Öffnung der Mauer; darauf nimmt eine Arbeitsgruppe von LINGUAPAX IV wie folgt Bezug (Centre UNESCO de Catalunya 1994: 21):

"In rather more than three years, from Saarbrücken (1990) to Barcelona (1994), the international situation has gone from hope for world

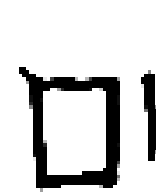
peace following the end oft the cold war to concern at the persistence and even increase of conflictivity in the emerging post-cold-war international system. Hence the title of the document summing up the results of the meeting (sc. in Saarbrücken; A.R.) and the attached proposals: 'For a culture of peace in a world without peace.'»

\section{LINGUAPAX und die Menschenrechte}

Die weltweiten Aktivitäten, die inhaltlich und weitgehend auch institutionell im Zusammenhang mit dem LINGUAPAX-Projekt stehen, lassen sich von verschiedenen Seiten beschreiben. Ein möglicher Weg wird von Prof. Kip Cates

(TottoriUniversity, Tottori, Japan) begangen. Erhat ein weit gespanntes Netz, nicht nur in Japan oder Ost-/Südostasien, sondern weltweit aufgebaut. Seine Aktivitäten betreffen die sog. «global issues»; sein Beitrag für unsere Veranstaltung in Saarbrücke enthält eine kurze Beschreibung dessen, was darunter zu verstehen ist (Cates 1993: 33):

"The goals of a 'global approach to education are generally divided into four domains of knowledge, skills, concern and action.

Knowledge about world problems 'out there' is the first goal. If we want our students to really work for a better world, then they must at least know the nature of world problems, their causes and viable solutions. They should also be aware of how world problems are related, how we as individuals are responsible for them and how solutions require changing unjust systems, not just wellmeaning individual actions. Acquiring skills necessary to solve world problems is the second goal. These are generally considered to include communication skills, critical and creative thinking, empathy, multiple-perspectives, co-operative problem solving, non-violent conflict resolution and informed decision-making. Concern is the third goal. With many of the world's problems perpetuated by selfishness, cynicism, apathy and despair, it is vital to help our students (and ourselves) break through these negative at- titudes to develop positive feelings of commitment and concern. Action is the final, most important, goal. When we know what the problems are, when we have the necessary skills and commitment to solve them, then we must take action and do what we can. Only in this way can we put an end to problems of war, injustice, hunger and environmental destruction.» Wir möchten im folgenden einen anderen Aspekt in den Mittelpunkt der Beschreibung rücken, der übrigens nach unserer Interpretation auch Teil des Gesamtfeldes «global issues» ist, nämlich den Zusammenhang zwischen Förderung des Friedens und Förderung der Menschenrechte, insbesondere der Sprachrechte in der Welt.

Die UNESCO hat das Projekt LINGUAPAX von Anfang an schwerpunktmäßig mit der Förderung der Menschenrechte in der Welt verbunden. Die Orientierung des Fremdsprachenunterrichts auf die Menschenrechte findet sich explizit in den Empfehlungen einer «Inter-Governemental Conference on Education in the Spirit of International Understanding, Cooperation and Peace and on Education in the Spirit of Respect for Human Rights and Fundamental Freedoms with a View to a Climate of Opinion Favourable to the Strengthening of Security and Disarmement», speziell in der Empfehlung Nr. 14 "Teaching of Modern Languages ${ }^{6}$

Grundlegende Aussagen zu den sprachlichen Rechten wurden 1987 in Recife/Brasilien auf einer Konferenz getroffen, die gemeinsam von der UNESCO und der AIMAV organisiert wurde Präsident dieses Kolloquiums ist eine Persönlichkeit, die sich in Südamerika in besonders herausragender Weise um die Durchsetzung der Menschenrechte im Verbund mit den Sprachrechten bemüht hat und bemüht: Prof. Francisco Gomes de Matos. Sein spezifisches Anliegen ist die Förderung des interkulturellen Diskurses als Grundvoraussetzung für die Verwirklichung der Rechte.

In der Abschlusserklärung heißt es u.a. (Skutnabb-Kangas \& Phillipson 1989:48):

- "Every social group has the right to positively identify with one or more languages and to have such identification accepted and respected by others.

- Every child has the right to learn the language(s) of his/her group fully. 
- Every person has the right to using the language(s) of his/her group in any official situation.

- Every person has the right to learn fully at least one of the official languages in the country where s/he is resident, according to her/his own choice.»

Dass die sprachlichen Rechte nicht nur geschützt, sondern überhaupt erst einmal hergestellt werden müssen, dass die Öffentlichkeit für diese Notwendigkeit sensibilisiert werden muss und dass die vorhandenen Rechtsauffassungen weiterentwickel werden müssen, war der Anlass für eine bedeutende und weltweit angelegte Veranstaltung, die von dem französischen Fremdsprachenlehrerverband (APLV) initiiert und von der FIPLV (Prof. Michel Candelier) mit Unterstützung der UNESCO durchgeführt wurde. Zeit und Ort dieser Veranstaltung waren symbolisch gewählt: Sie fand im Verbund mit den Etats Généraux des Langues (26. bis 29. April 1989) am 25. April 1989 in Paris statt, 200 Jahre nach der Französischen Revolution und der Verkündung der Menschenrechte 1789. Die dort verabschiedete Erklärung enthielt folgende Passagen, die den Weg von den allgemeinen Menschenrechten hin zum Recht auf Fremdsprachen aufzeigt; dieses Dokument wird damit zur wichtigsten Grundlage für weitere sprachenpolitische Aktivitäten ${ }^{7}$

«1. A l'occasion du bicentenaire de la Révolution Française et de la Déclaration des Droits de l'Homme et du Citoyen de 1789 , notre projet est d'obtenir que les Droits suivants soient reconnus explicitement par la Communauté Internationale et les différents Etats ou groupes d'Etats qui la composent a) Tout homme a le doit de se développer dans la langue et la culture du groupe d'origine auquel il appartient.

b) Tout homme a le droit de dépasser les limites de cette langue et de cette culture pour accéder à une diversité d'autres langues et d'autres cultures.

c) Toute Communauté a le devoir d'offrir à chacun la possibilité d'accéder à la plus grande diversité possible de langues et de cultures. Tout Homme a le droit de choisir librement les langues qu'il veut apprendre et les cultures auxquelles il veut s'ouvrir. "

2. Ces droits correspondent à diverses nécessités relatives au développement harmonieux des potentialités de chaque individu, à l'insertion la plus fructueuse de 1'individu dans la société moderne, à la Paix et à la coopération entre les communautés, à l'épanouissement des cultures dans leur diversité.

Pour a):

La langue et la culture d'origine représentent un apport irremplaçable pour un développement harmonieux des aptitudes intellectuelles et affectives, en particulier i l'époque de la première socialisation.

Pour b):
La communication de chaque individu-dans le travail ou les loisirs, pour la vie publique ou la vie privée-avec des membres d'autres groupes, d'un autre pays ou du même pays, est de plus en plus fréquemment un facteur indispensable d'efficacité professionnelle et de réalisation de sol; c'est un élément de la liberté d'expression;

- l'accès à la part la plus large possible de l'héritage scientifique et culturel de l'Humanité est un facteur d'enrichissement personnel et collectif dont personne ne devrait être privé. Il permet en retour une meilleure compréhension de soi-même et de ses propres valeurs;

- le recours à une autre langue permet à l'individu d'exprimer de façon plus complète ce qu'il ressent du monde et de lui-même, en se libérant du système de conventions linguistiques et culturelles qui constitue sa langue et sa culture d'origine.

Die Versammlung in Paris 1989 hat sich folgende Argumentationskette zu eigen gemacht, die ganz direkt auf das Recht, Fremdsprachen zu lernen, orientiert ist:

«La Déclaration des Droits de l'Homme proclame que l'éducation 'doit favoriser la compréhension, la tolérance et l'amitié entre toutes les nations et tous les groupes raciaux ou re- ligieux, ainsi que la développement des activités des Nations Unis pour le maintien de la paix'. La Déclaration des Droits de l'Enfant affirme que l'enfant 'doit être élevé dans un esprit de compréhension, de tolérance, d'amitié entre las peuples, de paix et de fraternité universelle'. L'accès à une diversité d'autres langues et d'autres cultures peut y contribuer de façon évidente.

Le droit à la vie culturelle en général est affirmé dans la Déclaration Universelle des Droits de l'Homme. Toute personne 'est fondée à obtenir la satisfaction des droits économiques, sociaux et culturels indispensables à sa dignité et au libre développement de sa personnalité'; 'Toute personne a le droit de prendre part librement à la vie culturelle de la communauté'».

Der Pariser Kongress 1989 folgert:

«La 'dignité' et la liberté des choix culturels qui sont ainsi revendiqués impliquent le respect de toutes les langues et cultures d'origine, et la possibilité, offerte par la société, d'accéder aux langues et aux cultures de son choix.»

\section{Aus Rechten werden Pflichten}

Von besonderem Interesse ist, dass aus all diesen Überlegungen und Quellen nicht nur Rechte folgern, sondern auch Pflichten; Fremdsprachen zu lernen ist eine Pflicht, denn nur auf diesem Wege kann der Einzelne zum Wohl der Gemeinschaft (des Staates, der Region) beitragen und nur dann kann der Einzelne die ihm zustehenden Rechte überhaupt einfordern.

Wir sind der Meinung, dass damit aber auch die entscheidende Grundlage dafür gegeben ist, dass die Gesellschaft zu einem angemessenen Angebot an Fremdsprachen verpflichtet wird. Der Europarat fördert mit seinen Projekten und Arbeiten die Mehrsprachigkeit in Europa in entscheidender Weise; ein Handbuch zu dieser Thematik ist im Jahre 2002 in Strassburg erschienen. Die Europäische Kommission vertritt die Forderung, dass jeder Bürger und jede Bürgerin Europas neben seiner Muttersprache/Erstsprache zwei Fremdsprachen lernen soll, und wir setzen hinzu: lernen können soll, d.h., die Möglichkeit dazu erhält. Wir setzen ferner hinzu, dass 
Mehrsprachigkeit erst dann realisiert wird, wenn die Bildungseinrichtungen verschiedene Sprachen anbieten, so dass aus der Plurilingualität des Einzelnen die Multilingualität der Gemeinschaft entstehen kann; dabei soll die Auswahl selbstverständlich über die Grenzen der europäischen Sprachen hinausgehen. "Sprachen als Brücken», Sprachen als Mittel des Verstehens und der Verständigung, Sprachen als Mittel zur Entfaltung der Persönlichkeit, Sprachen als Mittel zur Schaffung sozialer Kohäsion, Sprachen als Instrumente der Friedensförderung: Das sind Elemente einer «Philosophie», wie sie von LINGUAPAX ebenso vertreten werden wie von Verbänden (z.B. FIPLV, AIMAV) und Institutionen (z.B. Europarat, Europäische Kommission, UNESCO).

\section{LINGUAPAX-heute und in Zukunft}

Die zahlreichen Aktivitäten von LINGUAPAX der letzten Jahre werden koordiniert und intensiv vorangetrieben durch das Centre UNESCO de Barcelone. Der Homepage dieser Institution entnehmen wir folgende Einzelheiten, die zeigen sollen, welche Möglichkeiten die Spracheninteressierten haben, sich in dieses Projekt einzubringen.

«Linguapax est un projet international de l'UNESCO, qui vise à promouvoir la culture de la paix à travers l'éducation plurilingue, la protection du patrimoine linguistique et les conseils donnés aux États membres en matière de politique linguistique.

Le projet Linguapax est dirigé par le ComitéInternational Linguapax et coordonné par la Division des langues de l'UNESCO en coopération

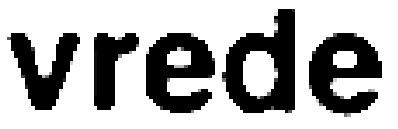

Les langues sont, par excellence, le véhicule du dialogue humain. La disparition des langues est une perte pour l'humanité tout entière. Pour ces raisons, le projet Linguapax a pour but de promouvoir la culture de la paix à travers l'éducation plurilingue et le respect de la diversité linguistique.»

Für die UNESCO ist das Programm LINGUAPAX eingebettet in den Bereich der education, ebenso wie die Sprachenprojekte des Europarats auch dort in die Abteilung education integriert sind ${ }^{11}$. Der Zusammenhang wird folgendermaßen gesehen:

«L'éducation est un élément essentiel du processus de socialisation des individus et, par

«Linguapax est un projet international de l'UNESCO, qui vise à promouvoir la culture de la paix à travers l'éducation plurilingue, la protection du patrimoine linguistique et les conseils donnés aux États membres en matière de politique linguistique.

Le projet Linguapax est dirigé par le Comité International Linguapax et coordonné par la Division des langues de l'UNESCO en coopération avec le Centre UNESCO de Catalogne.»

Fèlix Martí schrieb mir kürzlich u.a. die folgenden Zeilen, in denen er die derzeitigen Entwicklungen von LINGUAPAX skizziert: «Comme vous savez peut-être, dans le cadre de la restructuration mise en œuvre par son nouveau directeur général, l'UNESCO a dissout la division des langues après le départ de Joseph Poth à la retraite. Le terme de Linguapax n'est plus officiellement au programme de l'Organisation et nous sommes à présent dans une phase d'expectative... Nous avons bon espoir avec le Centre UNESCO de Catalogne.» Die Förderung der Mehrsprachigkeit wird von dem Projekt LINGUAPAX ebenso als Hauptaufgabe angesehen wie dies auch im Europarat der Fall ist. Diese Aktivitäten haben folgende Ziele:

«Toutes les langues sont égales en ce qu'elles constituent une interprétation de la réalité, de création culturelle et de cohésion sociale. Les conflits entre les peuples naissent des inégalités, des injustices et du manque de respect des identités culturelles, et non de la diversité linguistique. que les interventions de nombreux Etats membres, tant au Conseil Exécutif comme à la Conférence Générale (qui sont recueillies dans les documents officiels del'Organisation) se traduiront d'ici peu en une nouvelle politique en matière de langues notamment au Secteur de l'Education. Par ailleurs, la récente approbation de la déclaration universelle de l'UNESCO sur la diversité culturelle devrait entraîner dans son sillage un regain d'intérêt pour la question linguistique dans le secteur de la culture.

Le Centre UNESCO de la Catalogne a toujours été un partenaire fidèle de l'UNESCO en matière de langues, depuis la conférence de Kiev (1987) qui marquait la naissance de ce qui est devenu une philosophie. C'est pourquoi nous avons décidé de créer, il y a tout juste un an, l'Institut Linguapax qui se consacrera tout particulièrement à la promotion des politiques linguistiques favorables à la diversité. Le premier grand événement de l'Institut est le Congrès Mondial sur les Politiques Linguistiques qui se tiendra à Barcelone du 16 au 20 avril prochain (2002; A.R.).»

Um die Ziele, die LINGUAPAX erarbeitet hat und in die Öffentlichkeit trägt, didaktisch und sprachenpolitisch durchzusetzen, bedarf es dreierlei:

- Im Sinne der Ziele von LINGUAPAX konkrete Arbeit leisten, d.h.: Lern-/Lehrmaterialien erstellen, Lehr-/Lernmethoden erarbeiten13, Lern-/Lehrbedingungen schaffen, Curricula konzipieren, Ressourcen zur Verfügung stellen, ein öffentliches Bewusstsein schaffen.

- Die Grundsätze von LINGUAPAX weiterentwickeln, sie entsprechend der Veränderung der gesellschaftlichen Situation konzeptuell und politisch fortschreiben.

- Sich sprachenpolitisch engagieren und das sprachenpolitische Engagement in politisches Tun umsetzen, ganz im Sinne dessen, was der Europarat unter «European citizenship/citoyenneté européenne» versteht.

Für diese Umsetzung gelten nach meiner Auffassung Leitlinien, wie wir sie für Europa, insbesondere im Rahmen des Europarates, zu entwickeln versuchen (Raasch 2002).

«Politisches Handeln verstehen wir als Förderung der 'citoyenneté'/der 'citizenship', als Förderung sozialer Kohäsion und politischer Kohärenz, Förderung des Dialogs zwischen Wertesystemen und als Fortschreibung der Menschenrechtskonzepte (durch die Charte européenne des Droits de l'Homme). Wir brauchen den Aufbruch zu einer demokratisch, europäisch konzipierten Sprachenpolitik mit den zentralen Zielen der Förderung der Mehrsprachigkeit, der Förderung der Entfaltungsmöglichkeiten der individuellen Persönlichkeit als sozialer Aufgabe und der Optimierung des Standortes, und das heisst der Region als Etappe zu einem größer werdenden Europa.» 


\section{Anmerkungen}

1. Expertentreffen in Venedig, 6. - 7. Oktober 1989.

2. Vgl. Siguan, 1997.

3. UNESCO, Final Report, 1987, dact..

4. Discussion Document for the FIPLV General Assembly, Sopron, 16 September 1988.

5. Dieser Newsletter ist abonnierbar unter: kcates@fed.tottori-u.ac.jp

In der Ausgabe Nr. 45 ist übrigens ein Beitrag von Dennis Cunningham zum Thema «Promoting Linguistic Diversity for Peace» enthalten (p. 7).

6. Diese UNESCO-Konferenz fand vom 12. bis 20. April 1983 in Paris statt.; weitere Konferenzen zu dieser Thematik folgten in Moskau 1985, in Prag 1985, in Moskau 1985, in Sofia 1983.

7. Fédération Internationale des Professeurs de Langues Vivantes Colloque «Langues et Droits de l'Homme», Paris, 25 avril 1989, organisiert von Michel Candelier.

8. FIPLV World News No. 51, World News No. 17, May 1989, p.7

9. Vgl. oben den Absatz 1 ("Al'occasion du...».) 10. Hervorhebung durch A.R.

11. Conseil de l'Europe Division des Langues vivantes, Dir. Prof. Joseph Sheils.

12. Wer nach konkreten Beispielen für Lernmaterialien und für Unterrichtsmethoden sucht, sei verwiesen auf:

Ackermann, P (1976), Frieden - Friedensstrategien. Stuttgart.

Classen-Bauer, I.(1989), International Understanding through Foreign Language Teaching. Handbook for Foreign Language Teaching as a Means for International Understanding. Bonn, German Commission for UNESCO.

Ferner:

Grasa, R. \& Reig, D. (1996), LINGUAPAX: Vivimos en un solo mundo, unidad didáctica 1. Barcelone: Centre UNESCO de Catalunya.

Grasa, R. \& Reig, D. (1996), LINGUAPAX: We live in just one world: teaching, unit 1. Barcelone: Centre UNESCO de Catalunya.

Grasa, R. \& Reig, D. (1997), Mundu bakar batean bizi gara, unitate didaktikoa 1 . Bilbao: UNESCO Etxea.

Grasa, R. \& Reig, D. (1998), Linguapax. [Presentation du project en espagnol, catalan, galicien, basque, anglais et français], Barcelone

Grasa, R. \& Reig, D. (1998), Els drets de la terra. Quaderns Linguapax. Barcelone
Grasa, R. \& Reig, D. (1998), Conviure amb els altres. Quaderns Linguapax. Barcelone. Grasa, R. \& Reig, D. (1998), Imatges i estereotips. Quaderns Linguapax. Barcelone. Grasa, R. \& Reig, D. (1998), El restaurant del món. Quaderns Linguapax. Barcelone. Grasa, R. \& Reig, D. (1998) Vivim en un sol món. Quaderns Linguapax. Barcelone. Landesinstitut für Pädagogik und Medien (LPM) (1989), Friedenser-

ziehung in der Schule. Konzeption und Anrgungen für den Unterricht. Saarbrücken.

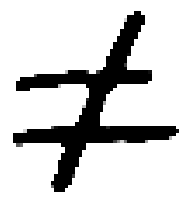
PS. «Oeuvrer pour la paix» ist der Titel des Thementeils in Französisch heute 33 (2002), Heft 3, koordiniert von Inez De Florio-Hansen.

\section{Bibliographische Hinweise}

Bressand,J.-M. (1995), Peace through Languages. Louvain.

Cates, K (1993), Teaching for a better world: Global Education and Foreign Language Teaching. In: Raasch 1993: 29 - 42.

Centre UNESCO de Catalunya (Hrsg.) (1994), LINGUAPAXIV. Barcelona, 24 - 27/II/1994. Barcelona.

Cunningham, D. \& Candelier, M. (Hrsg.) (1996), LINGUAPAX V. Melbourne - Australia, June 30 - July 2 1995. Belgrave.

Freudenstein, R. (1993), Integrating Peace into the Foreign Language Curriculum. In: Raasch 1993: 75 - 81.

Freudenstein, R. (1997), Peace Education? No, Thank You!. In: Raasch 1997: 84 - 94.

Grève, M. de (1991), Considérations épistologiques sur la notion de paix. In: Raasch 1991: 43-53.

Hummel, G. (1997), Komm näher, Frieden, komm...! In: Raasch 1997: 14-19.

Poth, J. (1997), Language planning in a plurilingual educational context (African edition). Guide pratique Linguapax no.1. Mons. Centre International de Phonétique Appliquée.

Poth, J. (1997), L'aménagement linguistique en contexte éducatif plurilingue (version Afrique). Guide pratique Linguapax no.1. Mons. Centre International de Phonétique Appliquée. Poth, J. (1997), La conception et la réalisation des manuels scolaires. Initiation aux techniques d'auteurs. Guide pratique Linguapax no.2. Mons: Centre International de Phonétique Appliquée.
Poth, J. (1997), L'enseignement des langues non maternelles. Une perspective psycho-pédagogique adaptée au contexte éducatif des pays plurilingues africains. Guide pratique Linguapax no. 3. Mons: Centre International de Phonétique Appliquée.

Poth, J. (1997), L'enseignement d'une langue maternelle et d'une langue non maternelle. La mise en application d'une pédagogie conver-

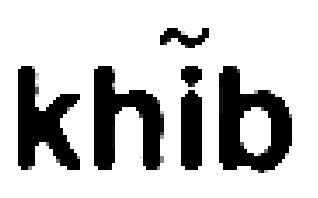
gente (version Afrique). Guide pratique Linguapaxno. 4. Mons: Centre International de Phonétique Appli-

quée.

Raasch, A. (Hrsg.) (1991), Peace through Language Teaching. La paix par l'enseignement des langues. Frieden durch Fremdsprachenunterricht. Saarbrücken.

Raasch, A. (Hrsg.) (1993), Language Teaching in a World without Peace. L'enseignement des langues dans un monde sans paix. Der Fremdsprachenunterricht in einer Welt ohne Frieden. Saarbrücken.

Raasch, A. (Hrsg.) (1997), Languages: Ways Towards Peace. Langues: Destination Paix. Sprachen: Wegezum Frieden. An Incentive for Language Teaching - for Peace. Un déclic pour l'enseignement des langues étrangères-pour la paix. Impulse für den Fremdsprachenunterricht - für den Frieden. Saarbrücken.

Raasch, A. (2002), Welche Sprachen im mehrsprachigen Europa? Internationale Sprachenkonferenz «Kleine und grosse Sprachen im (zusammen)wachsenden Europa», Helsinki, 9.11. 2001

Siguan, M. (1990) (coord.), Las lenguas y la educación para la paz. Linguapax II, XIII Seminario sobre «Educación y lenguas». Barcelone

Siguan, M. (2001), Die Sprachen im vereinten Europa. Aus dem Spanischen übersetzt von Silvia Parra Belmonte. Mit einem Geleitwort von Albert Raasch. Tübingen.

Simon-Pelanda, Hans (1997), Projekt Spurensuche: Friedenserziehung. In: Raasch 1997: 20-83.

Skutnabb-Kangas, T. \& Phillipson, R. (1989), Wanted! Linguistic Human Rights. Roskilde UniversitetsCenter Lingvistgruppen.

UNESCOLINGUAPAX (1997). [Seminaire International sur les Politiques Linguistiques, tenu à Leioa (Pays Basque) du 11 au 14 de mars 1996]. Bilbao: UNESCO Etxea.

Wimmer, R. \& Wimmer, W. (Hrsg.) (1987), Friedenszeugnisse aus vier Jahrtausenden. Leipzig/Jena/Berlin. 